\title{
Rotors in Polygons and Polyhedra
}

\section{By Michael Goldberg}

1. Introduction. Curves of constant width are closed curves which can be rotated through all orientations between two fixed parallel straight lines while remaining tangent to these lines. They are also known as curves of constant breadth, Gleichdicke (in German), or orbiformes (in French). They have been studied by mathematicians beginning with Euler [1], Minkowski [3], Blaschke [8, 9], Schilling [11], and others to the present time. They have been applied in mechanisms to generate a wide variety of periodic motions. They have been used as the shapes of drills for drilling square and hexagonal holes. They have been produced unintentionally in certain manufacturing processes when only precise circular cylinders were desired.

Two more fixed parallel lines may be added without constraining the rotation. In particular, the four lines may form a square. Hence, the curve may rotate while remaining tangent to all sides of the square. For this reason I call the curve a rotor in a square.

Immediately, there arises the question whether non-circular rotors exist for other polygons. It has been found that they exist for all regular polygons and various methods of deriving them have been developed. The earliest complete development was published in 1909 by Meissner, a Swiss mathematician [4]. He derived the rotors in the $n$-gons and described them analytically by means of the polar tangential equation

$$
p=a_{0}+\sum_{k=1}^{\infty}\left(a_{k} \cos k \theta+b_{k} \sin k \theta\right)
$$

where $p$ is the distance from the origin to the tangent to the curve, $\theta$ is the angle which the normal makes with the reference axis, the $a_{k}$ and $b_{k}$ are arbitrary constants except that

$$
a_{k}, b_{k}=0 \text { for } k \neq \pm 1(\bmod n) .
$$

(For example, if $n=6$, the terms which do not have to be zero are obtained for $k=1,5,7,11,13,17,19$, etc.) Hence, for each polygon there is an infinity of different rotors. For convexity, the constants $a_{k}$ and $b_{k}$ are limited by certain inequality relationships.

2. Circular-Arc Rotors. Special cases have received special attention. In particular, those rotors which are bounded entirely by arcs of circles have been considered. Euler considered the rotor made of three equal arcs, each centered on a vertex of an equilateral triangle. Reuleaux [2] considered rotors of an odd number of circular arcs of equal radii. They have been called Reuleaux polygons, but I prefer to call them Reuleaux rotors since they are not polygons. The regular Reuleaux rotors are rotors in a square.

Received March 23, 1960. Retiring Presidential Address, The Philosophical Society of Washington, delivered at the 1485 th meeting of the Society on January $8,1960$. 

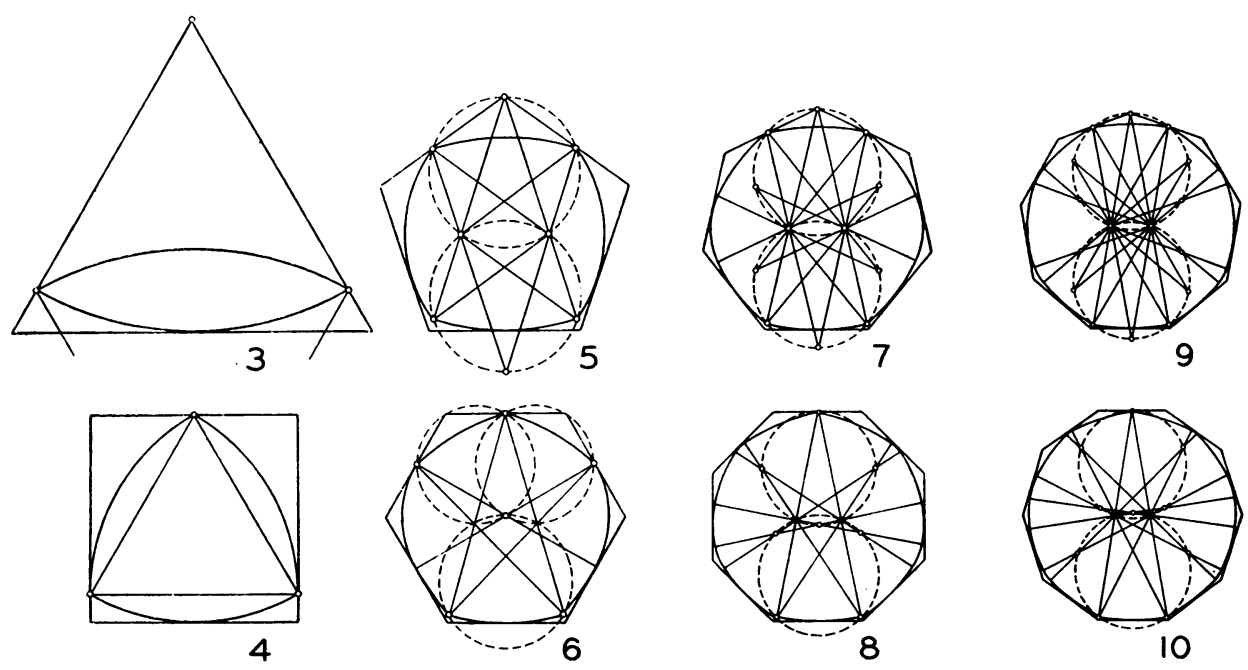

Fig. 1.-Circular-arc rotors in regular polygons.
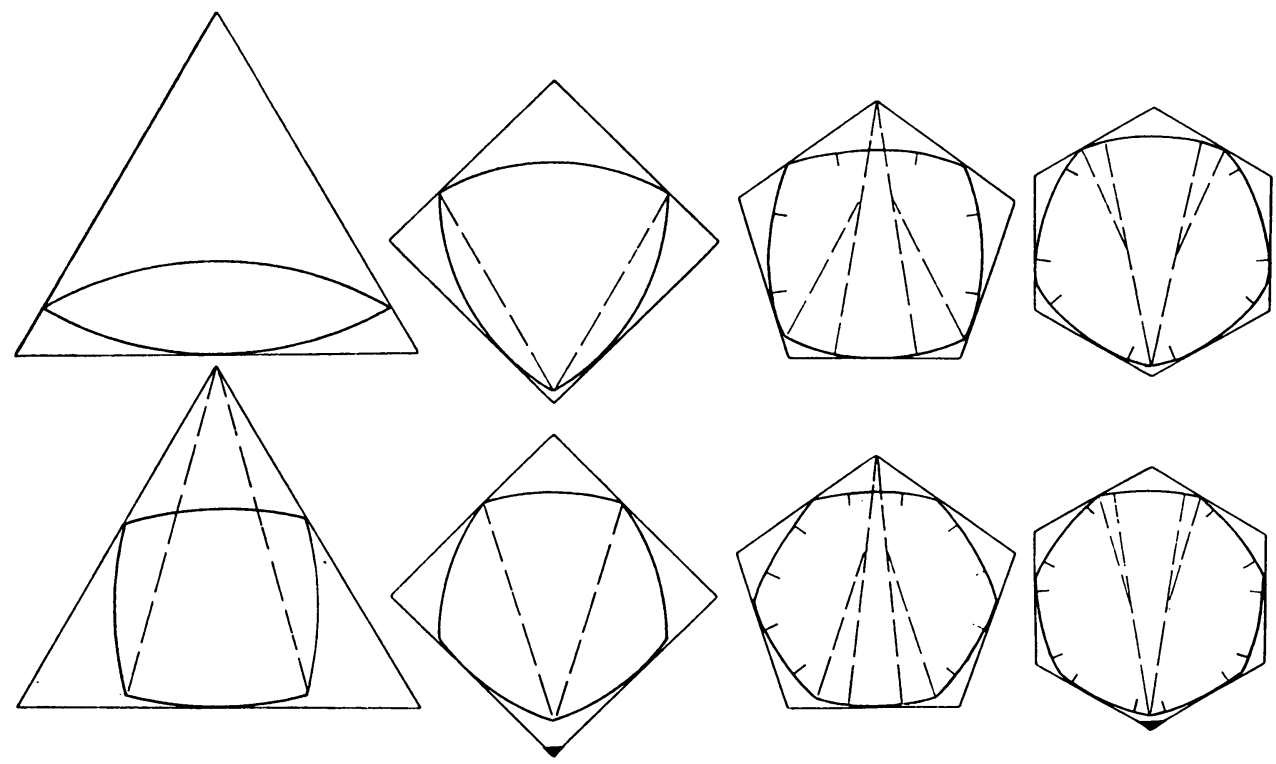

FIG. 2.-Regular trammel rotors in regular polygons.

Fujiwara [14] considered rotors in a triangle. He showed that some of the Reuleaux rotors are also rotors in a triangle. Besides these, he derived the rotors of two, four and five circular arcs. No such rotors of three arcs exist. Of the possible rotors of two arcs, one had already been published by Reuleaux [2]. A circulararc rotor for the pentagon is also described by Fujiwara [14, pp. 245-246]. In 1948, I published a paper describing the construction of circular-arc rotors for all the regular polygons [29]. The method is shown in Figure 1 where the centers of the arcs are regularly distributed on two generating circles, except for $n=6$ where 
three generating circles are used. Note that for $n>4$, the rotors for the even polygons possess only one axis of symmetry (unequal generating circles), while the rotors in the odd polygons possess two axes of symmetry (equal generating circles). In these, the radii of the arcs are not equal. In particular, note that the rotor in the pentagon is composed of two pairs of arcs, the radii of one pair being twice the radii of the other pair. It is conjectured that, among all the rotors whose contours are made of discrete circular arcs, these have the least number of arcs.

In 1957, I published a new series of circular-arc rotors which are characterized by higher orders of symmetry [32]. They are illustrated in Figure 2. These regular rotors in a regular $n$-gon are of two types. The upper series is made of $(n-1)$ equal segments. The lower series is made of $(n+1)$ equal segments. For $n>4$, each segment is made of three or more circular arcs, each arc being tangent to its neighboring arcs except at the ends of the segments.

3. Trammel Method of Construction. All the circular-arc rotors may be obtained kinematically by a graphical method which I call a trammel construction. This consists of moving the rotor so that, for each portion of the motion, two points of the rotor trace two fixed straight lines. In Figure 3, the rotor to be generated in the hexagon is based on a regular pentagon shown in dotted lines. The pentagon is turned counter-clockwise so that the vertices $B$ and $C$ move along the straight sides of the hexagon until the next vertex $D$ touches the hexagon. The motion is continued with vertices $C$ and $D$ moving along the sides of the hexagon. During these motions, the sides of the fixed hexagon will mold a rotor based on the dotted pentagon; that is, the rotor is the envelope of the sides of the hexagon on the plane of the moving pentagon.

4. Any Rotor as the Sum of Trammel Rotors. Every rotor in a $k n$-gon (where $k$ is any integer) is also a rotor in an $n$-gon. Hence, we have obtained an infinite number of circular-arc rotors for each polygon. Furthermore, if $p_{1}=f_{1}(\theta)$ and $p_{2}=f_{2}(\theta)$ are the polar tangential equations of two trammel rotors in a given polygon, then their weighted mean, given analytically as

$$
p_{3}=\left[u f_{1}(\theta)+v f_{2}(\theta)\right] /(u+v)
$$

where $u$ and $v$ are any real numbers, is the polar tangential equation of a new rotor in the same polygon. By extension, any rotor in a polygon can be expressed as a
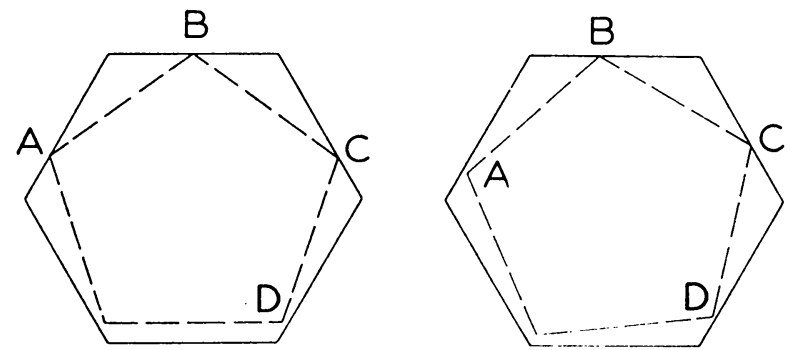

FIG. 3.-Vertices of regular $(n-1)$-lobed rotor in $n$-gon. 
weighted mean of a series (possibly infinite) of regular trammel rotors. This is similar to the Fourier series expansion used by Meissner as described in Section 1.

5. Basic Rotors and their Construction. In Meissner's equation, each variable term in the right-hand member can serve as the enture variable part of the righthand member of the equation for a rotor. These rotors, which I call basic rotors, resemble the regular circular-arc rotors [31]. However, the curvature of the arcs varies continuously instead of remaining constant by segments.

Each basic rotor can be obtained kinematically by the following method. Consider a fixed circle of circumference $c$. Compare with Figure 4. Let another circle of circumference $c n /(n+1)$, where $n$ is any integer, roll within the first circle without slipping. Then each point of the rolling circle describes a hypocycloid of $(n+1)$ cusps. Let a straight line be carried by the rolling circle. The envelope of the positions of this straight line will be a parallel curve of the hypocycloid. If the straight line is sufficiently distant from the center of the rolling circle, the envelope will be convex. This convex curve of $(n+1)$ maximal points or lobes is a basic rotor in a regular $n$-gon, as shown by the following argument.

When the center of the rolling circle returns to its initial position, the circle and the carried straight line will have undergone a rotation of $2 \pi / n$. As the rolling is repeated successively, there will be $n$ symmetric positions of the straight line. Therefore, the $n$ positions of the straight line will form a regular $n$-gon. The rolling circle can then be considered to carry the regular $n$-gon. As it rolls, the $n$ envelopes of the $n$ sides are the same. Therefore, each side of the $n$-gon keeps in contact with the envelope as the $n$-gon is rotated. Inversely, the $(n+1)$-lobed rotor can rotate continuously within the $n$-gon while keeping contact with all the sides of the $n$-gon.

A similar procedure obtains for $(n-1)$-lobed rotors in a regular $n$-gon.

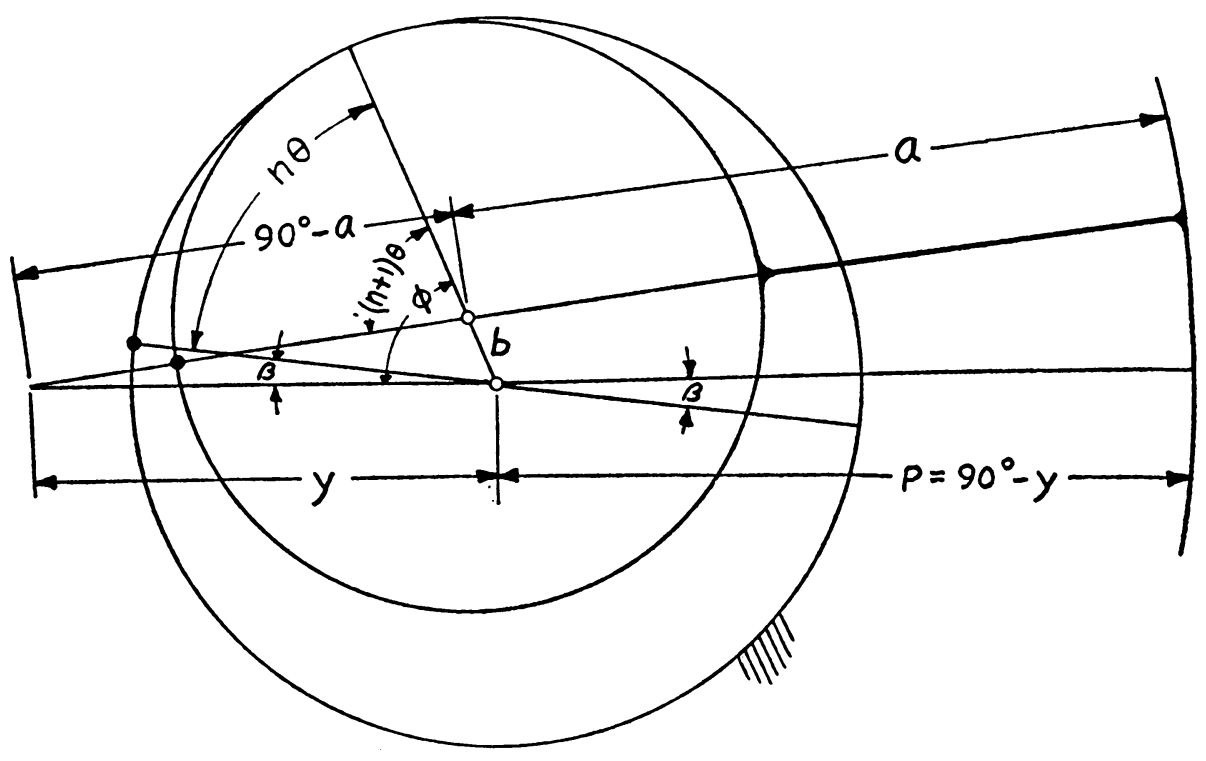

FIG. 4.-Generation of basic rotors. 


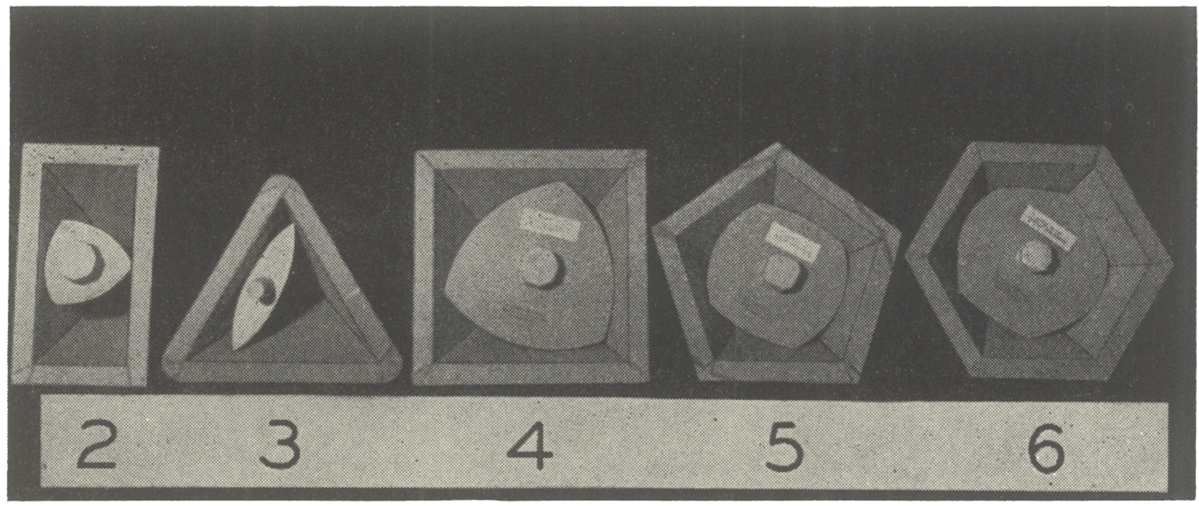

FIG. 5.-Conical rotors in pyramids

6. Rotors in Spherical Polygons. The concept of rotors has been generalized from the two dimensions of the plane to three and more dimensions. However, the intermediate case of rotors in spherical polygons has received very little attention. Blaschke [9] and Santaló [25] have considered ovals of constant width on the surface of the sphere. These are ovals which remain tangent to the two meridians which bound a line.

These were generalized by the author to include rotors in all the regular spherical polygons. The trammel rotor method of constructing circular-arc rotors in plane polygons is also applicable for the construction of rotors in spherical polygons. This time, however, the generated ares are not plane and, therefore, they are not circular [30].

Also, the spherical rotors corresponding to the basic plane rotors can be generated by the rolling circle method. In this case, the rolling circle carries an arc of a great circle instead of a straight line. (See Figure 4.) The symmetric positions of this great circle make a regular spherical polygon [31].

Every rotor in a plane polygon has its counterpart as a rotor in a spherical polygon. However, there are more types of spherical rotors for two reasons. The most obvious reason is that regular spherical polygons of the same number of sides are not similar; their shape depends upon their size. Therefore, their rotors are correspondingly different. But the more surprising difference between plane and spherical rotors is the fact that spherical ovals of constant width, which are tangent to two arcs of great circles, are distinct from rotors in a spherical quadrilateral. In the plane, these two sets of rotors are identical.

Models of spherical surfaces are difficult to construct and to demonstrate. However, every spherical polygon can be converted into a pyramid by passing planes through the great circles by which it is bounded. The rotor in the spherical polygon is replaced by a non-circular cone which is now a rotor in a pyramid. Models of several conical trammel rotors are shown in Figure 5.

7. Rotors Tangent to $n$ Circles. The trammel method and the rolling circle method may be applied in the derivation of other types of rotors. For example, $n$ equal circles may repla se the $n$ straight lines of a plane regular $n$-gon [33]. Among the interesting possibilities is a series of rotors which approximate regular polygons. 

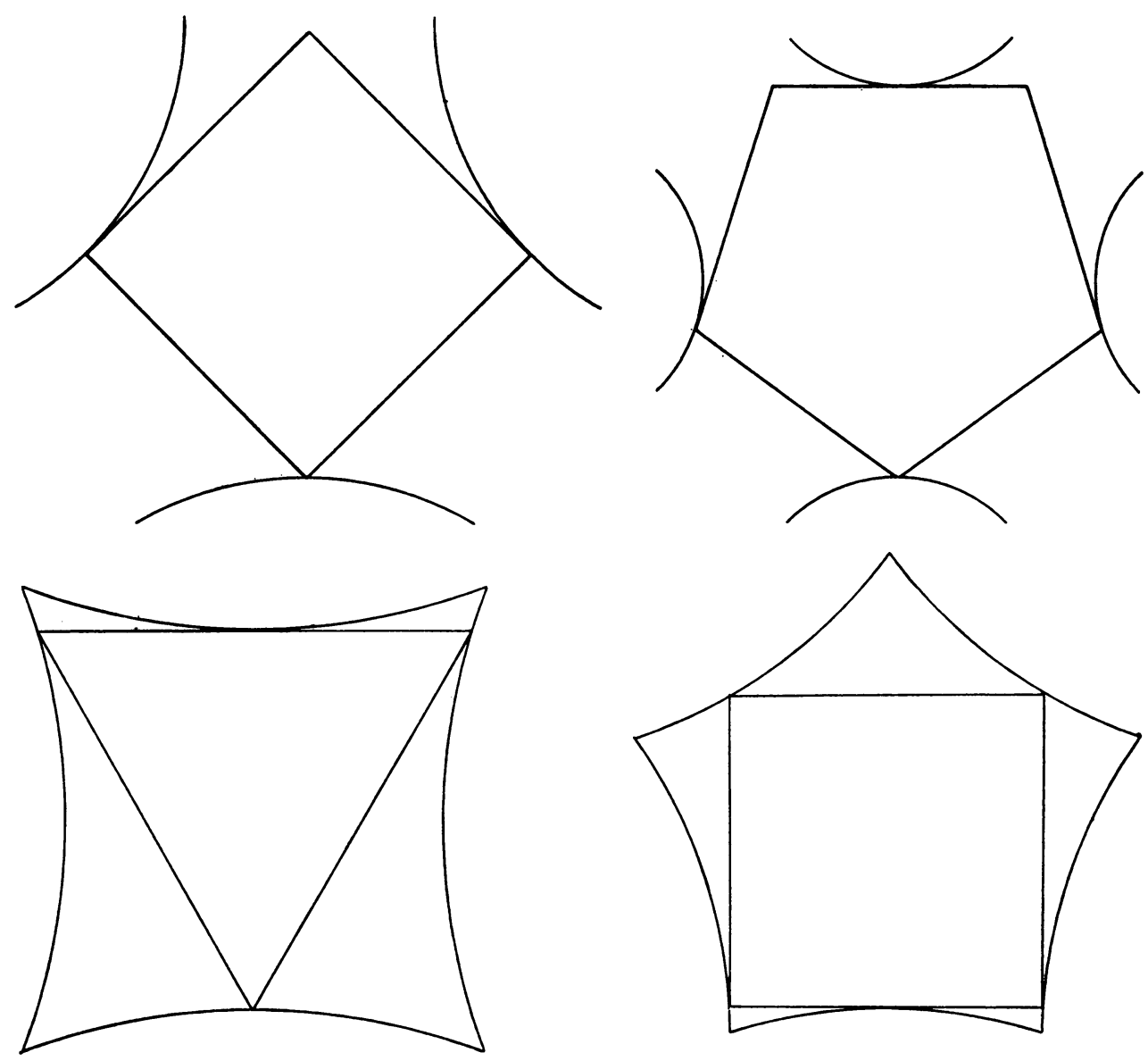

Fig. 6.-Rotors tangent to $n$ fixed circles.

Several are shown in Figure 6. Also, the methods are just as applicable on the surface of a sphere.

A further generalization is the use of a set of $n$ symmetric curves instead of $n$ circles. No special interesting cases are available at present.

8. Rotors in Regular Polyhedra. The first extensive study of surfaces of constant width was made by Minkowski [3]. He devised general geometric methods of deriving many of them. Many beautiful theorems concerning them were obtained. The most obvious method of generating one is by revolving a symmetric oval of constant width about its axis of symmetry. A more unusual surface is based on a regular tetrahedron [6]. Each vertex serves as a center of a spherical surface passing through the other vertices. However, one edge of each pair of opposite edges must be chamfered to a portion of a toroidal surface. The foregoing shapes are shown as $A$ and $B$ of Figure 7.

The surface of constant width is a rotor in a cube. The general investigation of rotors for all the regular polyhedra was first considered by Meissner. In a very 
elegant paper [7], he showed that non-spherical rotors exist for the regular tetrahedron, the cube and the regular octahedron, and that they do not exist for the regular dodecahedron (12 faces) and the regular icosahedron (20 faces). Just as Meissner used a Fourier series development for rotors in plane polygons, he used a spherical harmonic development for studying rotors in regular polyhedra. He showed that the rotors in the cube may be described by the polar tangential equation

$$
p(\theta, \phi)=a_{0}+Y_{1}+Y_{3}+Y_{5}+Y_{7}+\cdots ;
$$

the rotors in the tetrahedron may be written as

$$
p(\theta, \phi)=a_{0}+Y_{1}+Y_{2}+Y_{5} ;
$$

and the rotors in the octahedron may be written as

$$
p(\theta, \phi)=a_{0}+Y_{1}+Y_{5},
$$

where $Y_{i}$ (a function of $\theta$ and $\phi$ ) is the spherical surface harmonic of the $i$ th degree.

The number of arbitrary parameters in the equation for the rotor in the cube is infinite. The number of parameters for the rotor in the tetrahedron is eleven, while the number of parameters for the rotor in the octahedron is eight. Several rotors for the tetrahedron are shown in Figure 8. A rotor for the octahedron is shown on the extreme right of Figure 7.

Before leaving three dimensions, it may be fitting to consider other possibilities. It is conceivable that non-regular polyhedra may have non-spherical rotors. In particular, it is not known whether open-ended regular prisms can have non-

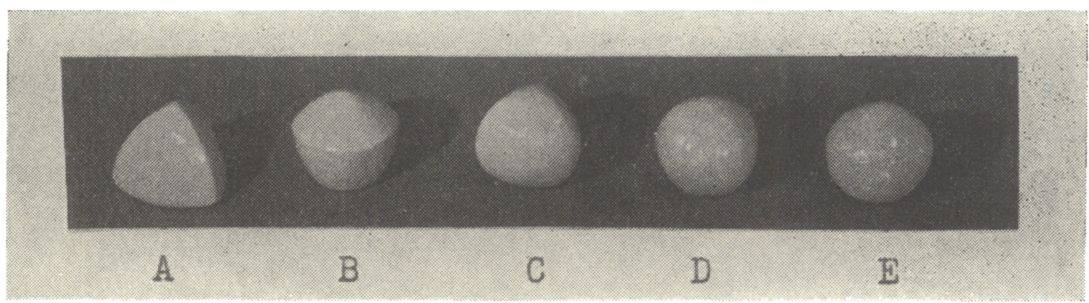

FIG. 7.-Rotors in cube.

A. Rotor based on tetrahedron; B. Rotor of revolution; C. Three-lobed rotor; D. Fivelobed rotor; E. Rotor in octahedron.

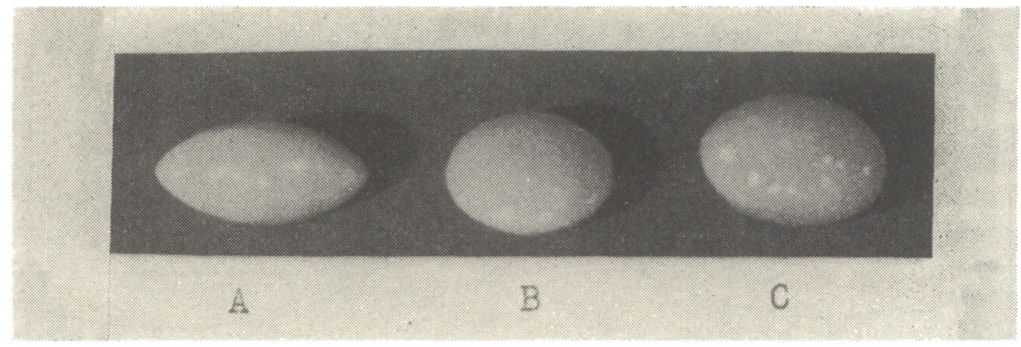

FIG. 8.-Rotors in regular tetrahedron.

A. Prolate rotor of revolution; B. Oblate rotor of revolution; C. Triaxial rotor. 
spherical rotors besides the well-known rotors in a rhombic prism which are surfaces of constant width.

9. Rotors in Higher Dimensions. Surfaces of constant width have been generalized to rotors in higher dimensions. Santalo [26] derived relations between measures of their boundaries and their contents as generalizations of relations derived by Minkowski [3, pp. 215-220]. Rotors in the simplex (the analogue of the triangle and the tetrahedron) also exist. Simple examples are the bodies of revolution given by the following polar tangential equations:

$$
\begin{array}{ll}
p=a+b \cos 2 \phi, & \text { Rotor in simplex, } \\
p=a+b \cos 3 \phi, & \text { Rotor in hypercube. }
\end{array}
$$

Non-spherical rotors for the analogue of the octahedron have not been mentioned in the literature.

10. Polygon Rotors in Ovals. If a rotor in a regular polygon is held fixed while the polygon is rotated about it, all the vertices of the polygon trace the same curve. Therefore, if this curve is fixed, the regular polygon can be rotated within it while all the vertices lie on the curve. Hence, for each rotor in a polygon, we have a curve within which the polygon can be rotated. See Figure 9. An application of a triangular rotor in an oval is a recent design, by the German engineer Felix Wanke!, of a non-reciprocating internal combustion engine. Test models of this engine have been made for the Curtiss-Wright Corporation.

This relation also applies for rotors in spherical polygons from which we obtain pyramidal rotors in non-circular cones.

However, in three dimensions, this relation does not apply. If a non-spherical rotor in a polyhedron is held fixed while the polyhedron is rotated, the vertices of the polyhedron do not lie on a surface. Instead, the positions of the vertices
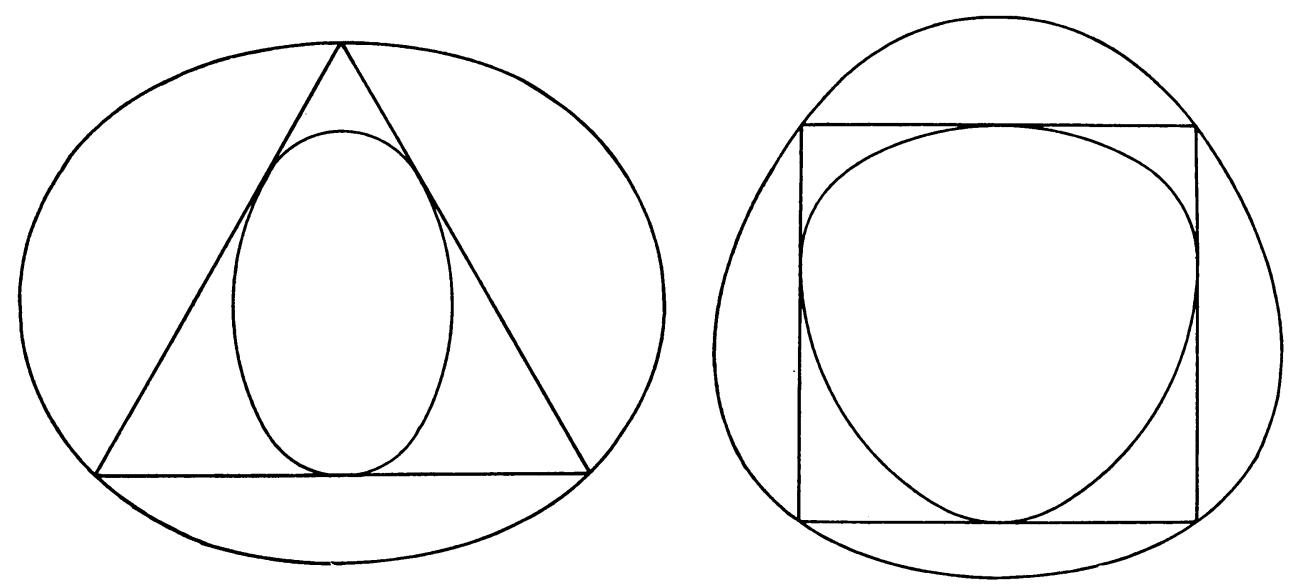

FIG. 9.-(a) Triangle rotor in an oval; (b) Square rotor in an oval. 


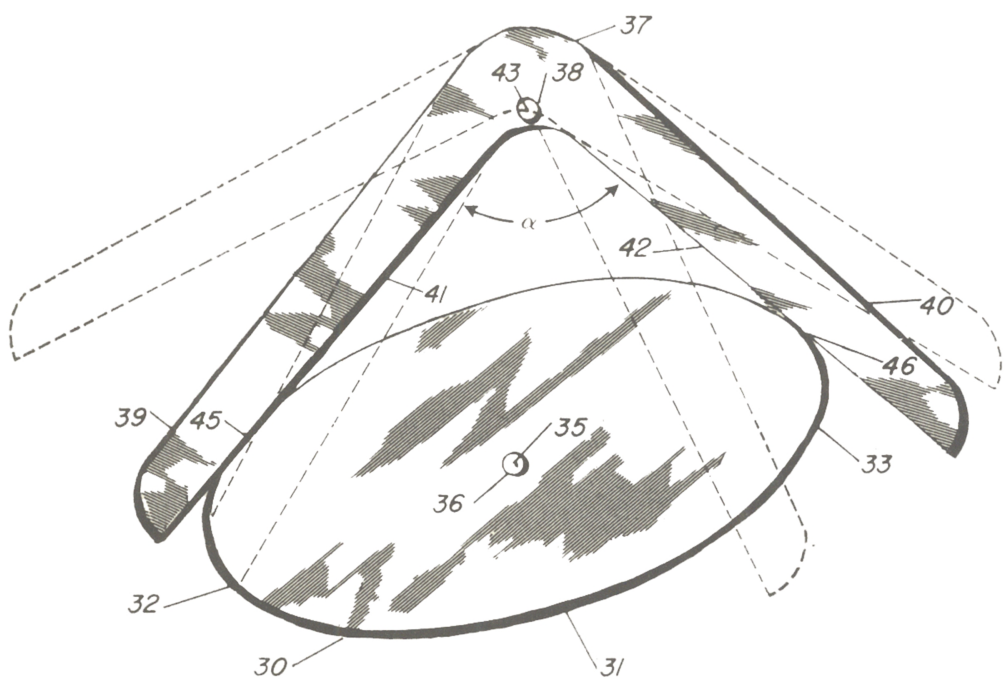

Fig. 10.-Double-contact cam

fill a volume bounded by two closed surfaces. The ellipsoid is the only known surface possessing the property that the vertices of all the circumscribing rectangular parallelepipeds lie on a sphere. It has been conjectured that every other body generates a volume in this manner and not a surface [10].

11. Mechanisms Related to Rotors. The use of drills in the shape of rotors in polygons has been extended from the drilling of even polygons (the square and the hexagon) to the drilling of odd polygons (the triangle and the pentagon). Several other problems, closely related to rotors in polygons, have been investigated. One is the determination of the non-circular shapes of pivoted rotors which remain in contact with two straight arms of a pivoted rocker arm. See Figure 10. Again, the only admissible angles between the arms are rational fractions of a circle as are the angles of a regular polygon. Examples of the ovals are described by

$$
p_{1}(\theta)=\cos p_{2}(\theta)
$$

where $p_{2}(\theta)=m \pi / 2 n+k \sin n \theta$, which is a rotor in a polygon. A geometer would describe these ovals as curves whose isoptic curves are circles [28]. They are the basis of a patent issued for a series of double-contact cam mechanisms [36].

Another related mechanism is the intermittent rotor [34]. This makes contact with a series of fixed elements but not always with all the elements. In the example shown in Figure 11, the rotor is restrained in its motion by contact with three of the four fixed points until all four of the fixed points are touched. The motion may then be continued with another set of three fixed points as constraints.

The presentation of new and unfamiliar basic mechanisms in this paper shows that the ancient science of mechanisms is far from exhausted. When the engineer 


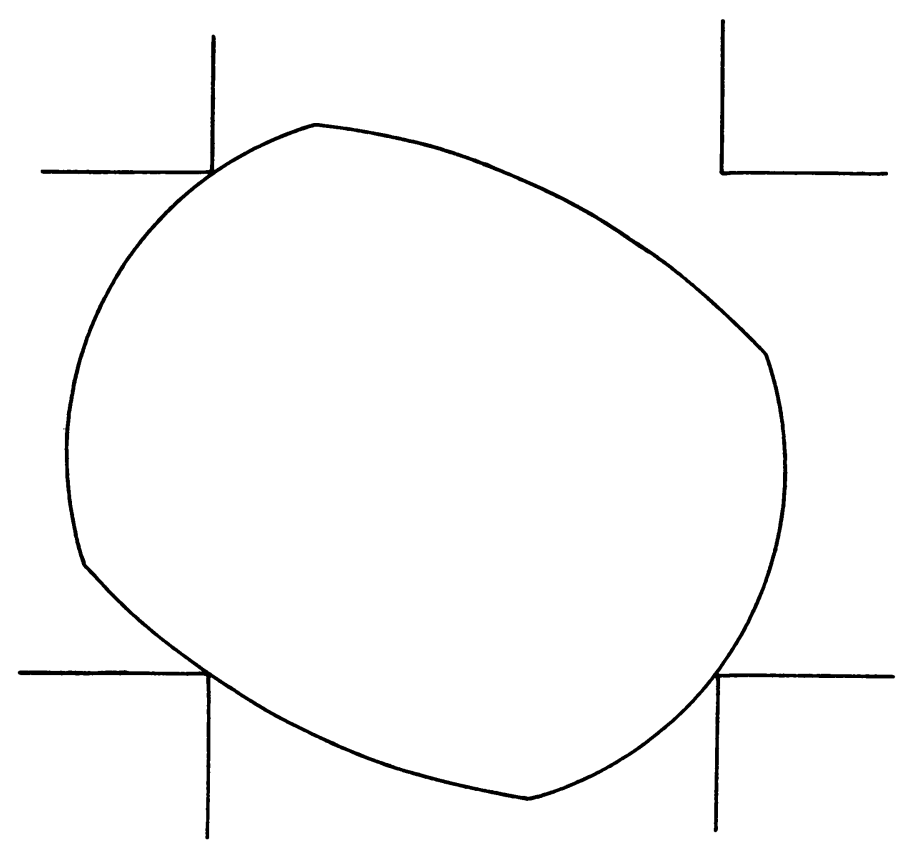

FIG. 11.--Intermittent rotor.

and designer become more familiar with them, it is expected that more applications will be made.

Bureau of Naval Weapons

Department of the Navy

Washington 25, District of Columbia

1. L. Euler, "De curvis triangularibus," Acta Acad. Sci. Petropolitanea, v. 2, 1778, p. 3-30.

2. F. Reuleaux, Theoretische Kinematik, 1875 .

3. H. MinkowskI, "Utber die Körper konstanter Breite," Rec. Math., Soc. Math. Moscow, v. 25, 1904, p. 505-508; Collected Works, v. 2, p. 277-279, 215-230.

4. E. Meissner, "Utber die Anwendung von Fourier-Reihen auf einige Aufgaben der Geometrie und Kinematik," Vierteljahrschrift der Naturforschenden Gesellschaft, Zürich, v. 54, 1909 , p. 309-329.

5. E. Meissner, "Utber Punktmengen konstanter Breite," Vierteljahrschrift der naturforschenden Gesellschaft, Zürich, v. 56, 1911, p. 42-50.

6. E. MEISSNER, "Drei Gipsmodelle von Flächen konstanter Breite," Zeitschrift für Mathematik und Physik, v. 60, 1912, p. 92-94.

7. E. MeISSNER, "Über die durch reguläre Polyeder nicht stützbaren Körper," Vierteljahrschrift der Naturforschenden Gesellschaft, Zürich, v. 63, 1918, p. 544-551.

8. W. BLASCHKE, "Konvexe Bereiche gegebener konstanter Breite und kleinsten Inhalts," Mathematische Annalen, v. 76, 1915, p. 504-513.

9. W. BLASCHKE, "Einige Bemerkungen über Kurven und Flächen von konstanter Breite," Ber., Verh. Sächs. Akad., Leipzig, v. 67, 1915, p. 290-297.

10. W. Blasches, Kreis und Kugel, Leipzig, 1916, p. 160.

11. F. Schilling, "Die Theorie und Konstruktion der Kurven konstanter Breite," Zeitschrift für Mathematik und Physik, v. 63, 1914, p. 67-136.

12. T. HAYASHI, "On the curves of constant breadth, and the convex closed curves inscribable and revolvable in a regular polygon," Science Reports, Tôhoku University, v. 5, 1916, p. 303-312.

13. M. FUJIWARA, "Utber die einem Vielecke eingeschriebenen und umdrehbaren konvexen geschlossenen Kurven," Science Reports, Tôhoku University, v. 4, 1915, p. 43-55.

14. M. FujIWARA, "Úber die innen-umdrehbare Kurve eines Vielecks," Science Reports, Tôhoku University, v. 8, 1919, p. 221-246.

15. M. FuJIWARA, "Analytical proof of Blaschke's theorem on the curve of constant breadth with minimum area," Proc., Tokyo Imp. Acad. Japan, v. 3, 1927, p. 307-309; v. 7, 1931, p. 300-302. 
16. M. FujIwARA \& S. KaKeYA, "On some problems of maxima and minima for the curve of constant breadth and the in-revolvable curve of the equilateral triangle," Tohoku Mathematical Journal, v. 11, 1917, p. 92-110.

17. G. TIERCY, "Sur les courbes orbiformes. Leur utilisation en mecánique," Tóhoku Mathematical Journal, v. 18, 1920, p. 90-115.

18. G. TIERCY, "Sur les surfaces sphériformes," Tóhoku Mathematical Journal, v. 19, 1921 , p. 149-163.

19. G. TIERCY, "Sur une transformation de mouvement circulaire en mouvement rectiligne alternatif (and other titles)," Comptes Rendu des Séances de la Société de Physique et d'Histoire Naturelle de Geneve, v. 40, 1923, p. 106-111, 128-130; v. 41, 1924, p. 38-43, $136-138$

20. H. BüCKNER, “Utber Flächen von fester Breite," Jahresbericht der Deutsche Mathematiker-Vereinigung, v. 46, 1936, p. 96-139.

21. H. Görtler, "Erzeugung stützbarer Bereiche," Deutsche Mathematik, v. 2, 1937, p. $454-466$; v. 3, 1938, p. 189-200.

22. W. WUNDERLICH, "Úber eine Klasse zwangläufiger höherer Elementenpaare," $Z$. Angew. Math. Mech., v. 19, 1939, p. 177-181.

23. H. Gericke, "Ưber stützbare Flächen und ihre Entwicklung nach Kugelfunktionen," Mathematische Zeitschrift, v. 46, 1940, p. 55-61.

24. H. Gericke, "Stützbare Bereiche in komplexer Fourier-Darstellung," Deutsche Mathematik, v. 5, 1940-41, p. 279-299.

25. L. A. Santal6, "Note on convex spherical curves," Bull., Amer. Math. Soc., v. 50, 1944 , p. 528-534.

26. L. A. Santal6, “On the convex curves of constant width in $E_{n}$," Portugaliae Mathematica, v. 5, 1946, p. 195-201, in Spanish.

27. L. A. Santal6, "Dos propiedades caracteristícas de los circulos sobre la superficie esférica," Mathematicae Notae, v. 11, 1951, p. 73-78.

28. J. W. Green, "Sets subtending a constant angle on a circle," Duke Math. Jn., v. 17, 1950 , p. 263-267.

29. M. GoldBerg, "Circular-arc rotors in regular polygons," Amer. Math. Mon., v. 55, 1948 , p. 393-402. 235-244.

30. M. GoldBerg, "Rotors in spherical polygons," Jn. Math. and Phys., v. 30, 1953, p.

31. M. Goldberg, "Basic rotors in spherical polygons," Jn. Math. and Phys., v. 34, 1956, p. $322-327$.

32. M. Goldberg, "Trammel rotors in regular polygons," Amer. Math. Mon., v. 64, 1957, p. $71-78$.

33. M. Goldberg, "Rotors tangent to $n$ fixed circles," Jn. Math. and Phys., v. 37, 1958, p. $69-74$.

34. M. Goldberg, “Intermittent rotors," Jn. Math. and Phys., v. 38, 1959, p. 135-140.

35. M. GoldBerg, "Method of measuring out-of-roundness of machined parts," U. S. Patent No. 2,515,214, July 18, 1950 .

36. M. Goldberg, "Double-contact cam mechanisms," U. S. Patent No. 2,741,132, April $10,1956$. 